OPEN ACCESS

Edited by:

Jianxun Ding,

Changchun Institute of Applied Chemistry, Chinese Academy

of Sciences, China

Reviewed by:

Ming Zeng,

First Affiliated Hospital of Jinan

University, China

Shengcai Yang,

Institute of Bioengineering and Nanotechnology (A*STAR),

Singapore

*Correspondence:

Yue Zheng

benbenzhu-11@163.com

Wei Lai

drlaiwei@163.com

${ }^{\dagger}$ These authors have contributed equally to this work

Specialty section:

This article was submitted to Biomaterials,

a section of the journal Frontiers in Bioengineering and

Biotechnology

Received: 02 November 2020 Accepted: 31 December 2020

Published: 11 February 2021

Citation: Zeng X, Peng M, Liu G, Huang Y, Zhang T, Wen J, Lai $W$ and Zheng $Y$ (2021) Strain Distribution and Drug Susceptibility of Invasive Fungal Infection in Clinical Patients With

Systemic Internal Diseases.

Front. Bioeng. Biotechnol. 8:625024. doi: 10.3389/fbioe.2020.625024

\section{Strain Distribution and Drug Susceptibility of Invasive Fungal Infection in Clinical Patients With Systemic Internal Diseases}

\author{
Xuehua Zeng ${ }^{1 \dagger}$, Mengran Peng ${ }^{2 \dagger}$, Guirong Liu' ${ }^{1}$, Yongqing Huang ${ }^{1}$, Tingting Zhang ${ }^{4}$, \\ Jing Wen ${ }^{3}$, Wei Lai ${ }^{2,3 *}$ and Yue Zheng ${ }^{2,3 *}$ \\ ${ }^{1}$ Clinical Laboratory, The Third Affiliated Hospital of Sun Yat-sen University-Yuedong Hospital, Meizhou, China, ${ }^{2}$ Department \\ of Dermatology, The Third Affiliated Hospital of Sun Yat-sen University, Guangzhou, China, ${ }^{3}$ Department of Dermatology, \\ The Third Affiliated Hospital of Sun Yat-sen University-Yuedong Hospital, Meizhou, China, ${ }^{4}$ Department of Traditional \\ Chinese Medicine, The Third Affiliated Hospital of Sun Yat-sen University-Yuedong Hospital, Meizhou, China
}

Background: Patients with systemic internal diseases present high risks for invasive fungal infections, which results in increased morbidity and mortality. Identification of high-risk departments and susceptibility systems could help to reduce the infective rate clinically. Correct selection of sensitive anti-fungal drugs not only could improve the cure rate but also could reduce the adverse reactions and complications caused by long-term antifungal drug treatment, which can be especially important in patients with serious systemic diseases. Therefore, the distribution changes of invasive fungal strains in patients with systemic internal diseases and the choice of antifungal drugs in clinical practice should be updated.

Objective: This work aimed to investigate the incidence, strain distributions, and drug susceptibility of invasive fungal strains isolated from patients with systemic internal diseases.

Methods: Samples were collected from 9,430 patients who were diagnosed with internal diseases in our hospital from January to December 2018. We then cultured and identified the fungal strains using API 20C AUX. We performed drug sensitivity analysis via the ATB Fungus-3 fungal susceptibility strip. Resistance was defined using the revised Clinical Laboratory Standardization Committee of United States breakpoints/epidemiological cutoff values to assign susceptibility or wild-type status to systemic antifungal agents.

Results: A total of 179 patients (49 female, 130 male) with fungal infection were included. The high-incidence departments were determined to be the respiratory department (34.64\%), intensive care unit (ICU; 21.79\%), and hepatology department (9.50\%). The susceptible systems for infection were the respiratory tract (sputum, $68.72 \%, 123 / 179$; secretion retained in the tracheal catheter, 3.35\%,6/179), urinary tract (urine, 9.50\%, 17/179), and gastrointestinal tract (feces, 9.50\%, 17/179). The major pathogens were Candida (90.50\%), Aspergillus (8.93\%), and Cryptococcus neoformans (0.56\%). The infective candida subgroups were Candida albicans (70.95\%), Candida 
krusei (6.15\%), Candida glabrata (5.59\%), Candida parapsilosis (3.91\%), and Candida tropicalis (3.91\%). The susceptibility of non-Aspergillus fungi for amphotericin B was $100.0 \%$. The susceptibility rates of 5 -fluorocytocine (5-FC) and voriconazole were 72.73 and $81.82 \%$, respectively, for C. krusei, 98.43 and 100\% for C. albicans, and $100 \%$ for both drugs for C. glabrata, C. parapsilosis, and C. tropicalis. The susceptibility rates of fluconazole and itraconazole were 0 and $54.55 \%$, respectively, for C. krusei, 20 and 20\% for C. glabrata, and 57.14 and $57.14 \%$ for C. tropicalis. The resistance rate of C. tropicalis for both fluconazole and itraconazole was $41.43 \%$.

Conclusion: Patients in the respiratory department, ICU, and hepatology department presented high rates of invasive fungal infections and should include special attention during clinical treatment. The respiratory tract, urinary tract, and gastrointestinal tract were the susceptible systems. Candida, especially C. albicans, was the main pathogen. From the perspective of drug sensitivity, amphotericin B should be given priority in treating the non-Aspergillus fungi infection in patients with systemic internal diseases, while the susceptibility of invasive fungal strains to azoles was variant. These data might provide clinical evidence for the prevention and treatment of invasive fungal infection in patients with systemic internal diseases.

Keywords: invasive fungal infection, epidemiology, drug susceptibility, drug resistance, systemic internal diseases

\section{INTRODUCTION}

Patients with systemic internal diseases present an increasing risk of invasive fungal infection. In recent years, the extensive use of broad-spectrum antibiotics, hormones, immunosuppressants, and other drugs used for the treatment of patients with internal diseases has given rise to an increase in the rate of invasive fungal infection. Furthermore, the development and extension of new technologies for organ transplantation and other procedures might also induce infections from conditional pathogens, especially fungal infections, as well as increase the likelihood to confer drug resistance changes (Bonduel et al., 2001). Identification of high-risk departments and susceptibility systems which are prone to suffer from invasive fungal infection could help reduce the clinical infective rate.

The drug sensitivity and resistance of invasive fungi to the frequent use of fluconazole and itraconazole in clinical treatment are changing. Some of the mechanisms for antifungal drug resistance include drug absorption and drug accumulation, decreased affinity of the drug to its target, alteration of metabolic pathways to disturb cellular drug concentrations, and biofilm formation (Ramana et al., 2013). Factors related to a patient's clinical situation and present co-morbidities, local epidemiology data, and purpose of treatment (prophylactic, preemptive, empiric, or definitive) should be taken into account when choosing the appropriate antifungal agents (Paramythiotou et al., 2014).

For the distribution of invasive fungi and drug sensitivity are varied, clinicians should select appropriate treatment schemes according to the patient's condition and the drug sensitivity results. Clinical awareness and knowledge of local epidemiology and pharmaceutical considerations could also help to achieve early diagnosis and treatment. Correct selection of sensitive antifungal drugs could not only improve the cure rate but also could reduce adverse reactions and complications caused by long-term antifungal drug treatment, especially in patients with serious systemic diseases. Therefore, knowledge of the susceptibility and resistance of invasive fungi strains to antifungal agents should be updated, especially in patients with systemic internal diseases who have received therapeutic drugs.

In this study, we investigated the incidence, strain distributions, and drug susceptibility of invasive fungal strains isolated from patients with systemic internal diseases in the southern area of China.

\section{MATERIALS AND METHODS}

\section{Fungal Strain Collection}

Both outpatient and inpatient samples, including sputum, urine, feces, blood, bile, cerebrospinal fluid, and secretion retained in the tracheal catheter, were collected from January to December 2018 at the Third Affiliated Hospital of Sun Yat-sen UniversityYuedong Hospital. The samples were collected from 9,430 patients who were diagnosed with internal diseases, and a total of 179 strains of invasive fungi were isolated.

\section{Ethics}

This work was an antifungal susceptibility surveillance study, and no human rights issues were involved. We obtained these strains in anonymized and de-identified forms. 


\section{Instruments and Reagents}

The Candida chromogenic culture medium was purchased from Jiangmen Kailin Trading Co., Ltd. We used the API 20C AUX identification instrument and ATB Fungus-3 fungal susceptibility strip by the French biological company Merieux.

\section{Culture and Identification}

The cultures were incubated in Sabouraud media and maintained at $35^{\circ} \mathrm{C}$ for $24-48 \mathrm{~h}$ in order to grow into a yeast-like colony. The single colony was then inoculated into Candida chromogenic culture medium and identified with an API 20C-AUX system.

\section{Susceptibility Testing}

We carried out the susceptibility test using the ATB Fungus 3 fungal susceptibility strip. Testing of the drug sensitivity of the minimum inhibitory concentration was obtained by utilizing ATB instrument interpretation. The strains were classified as sensitive, intermediate, or drug-resistant according to the Clinical Laboratory Standardization Committee of United States standard and ATB Fungus 3 product specification. The instructions were observed in detail, and the results were read with the naked eye if necessary. The quality control strain used was Candida albicans, ATCC 90028.

\section{Statistical Analysis}

Data processing and statistical analysis were performed using SPSS software (version 16.0, Inc., Chicago, IL, United States). Count data variables were expressed as frequencies and percentages. For all statistical analyses, statistical significance was accepted at $P<0.05$ (two-sided).

\section{RESULTS}

\section{High-Incidence Departments}

A total of 179 fungal infection patients (49 females, 130 males) were included. Of those, $34.64 \%$ patients $(n=62)$ were in the respiratory department, $21.79 \%$ patients $(n=39)$ were in the intensive care unit (ICU), and $9.50 \%$ patients $(n=17)$ were in the hepatology department (Figure 1).

\section{Susceptibility System Analysis}

The susceptible systems for infection were the respiratory tract, urinary tract, and gastrointestinal tract. Strains of fungi were isolated from the infective tissues or secretions of these patients, with $68.72 \%(123 / 179)$ coming from sputum, 9.50\% (17/179) from urine, and 9.50\% (17/179) from feces (Table 1).

\section{Analysis of Fungal Species in Clinical Infection}

The invasive fungi isolated were primarily Candida, which accounted for $90.50 \%$, with the remaining being Aspergillus, which accounted for 8.93\%, and Cryptococcus neoformans, which accounted for $0.56 \%$. Among the Candida species, Candida albicans accounted for $70.95 \%$, Candida krusei accounted for $6.15 \%$, Candida glabrata accounted for $5.59 \%$, Candida parapsilosis accounted for $3.91 \%$, and Candida tropicalis accounted for $3.91 \%$.

\section{In vitro Susceptibilities Among Non-Aspergillus Fungi}

Of the 179 invasive fungi detected, we analyzed the susceptibility of 163 strains of non-Aspergillus fungi to five kinds of antifungal drugs. The results showed that the drug susceptibility rate of the non-Aspergillus fungi for amphotericin B was $100.0 \%$. The susceptibility rates for 5-FC and voriconazole were, respectively, 72.73 and $81.82 \%$ for C. krusei, 98.43 and $100 \%$ for C. albicans, and $100 \%$ for both drugs for C. glabrata, C. parapsilosis, and $C$. tropicalis. The susceptibility rates of fluconazole and itraconazole were, respectively, 0 and $54.55 \%$ for C. krusei, 20 and $20 \%$ for C. glabrata, and 57.14 and $57.14 \%$ for C. tropicalis. The resistance rate of $C$. tropicalis for both fluconazole and itraconazole was $41.43 \%$ (Table 2).

\section{DISCUSSION}

Invasive fungal infections have been associated with increased morbidity and mortality, and the number of patients at risk of suffering invasive fungal infection is increasing (IbáñezMartínez et al., 2017). Several factors can contribute to this effect, and they include the widespread adoption of aggressive immunosuppressive therapy (e.g., chemotherapy, transplants), the use of new immune-modifying drugs among certain patient populations, and the increasing use of invasive devices such as central venous catheters (Enoch et al., 2017).

Our data have indicated that the clinical specimens of invasive fungi were mainly from a few departments, the respiratory department, the ICU, and the hepatology department, from which the composition breakdown was $34.64,21.79$, and $9.50 \%$, respectively. We can conclude from our results that patients in the departments that presented high rates of invasive fungal infections, specifically the respiratory department, ICU, and hepatology department, should include special attention during clinical treatment. Patients in the ICU often have a serious illness, are committed to long-term stays in the hospital, and are subjected to extensive use of antibiotics, hormones, or immunosuppressants and invasive operations, all of which could increase the susceptibility of secondary fungal infection. Bassetti et al. (2017) suggested that further investigation was needed to determine the incidence of invasive aspergillosis in the ICU, its relationship with influenza outbreaks, the clinical impact of rapid diagnosis, and the significance of combination treatment.

Previous studies have revealed that fungal infections were identified primarily in the respiratory tract (Smith and Kauffman, 2012) and urinary tract. Furthermore, $86.03 \%$ of infective patients were persons over 60 years old who had weak mucociliary clearance ability and more bronchial gland hyperplasia and secretion (Anaissie et al., 2009). We found that, besides the respiratory tract and urinary tract, the gastrointestinal tract was also a susceptible system in which deep fungal infection appeared in patients with systemic diseases. 


\section{Distribution and composition of Fungi from clinic department}

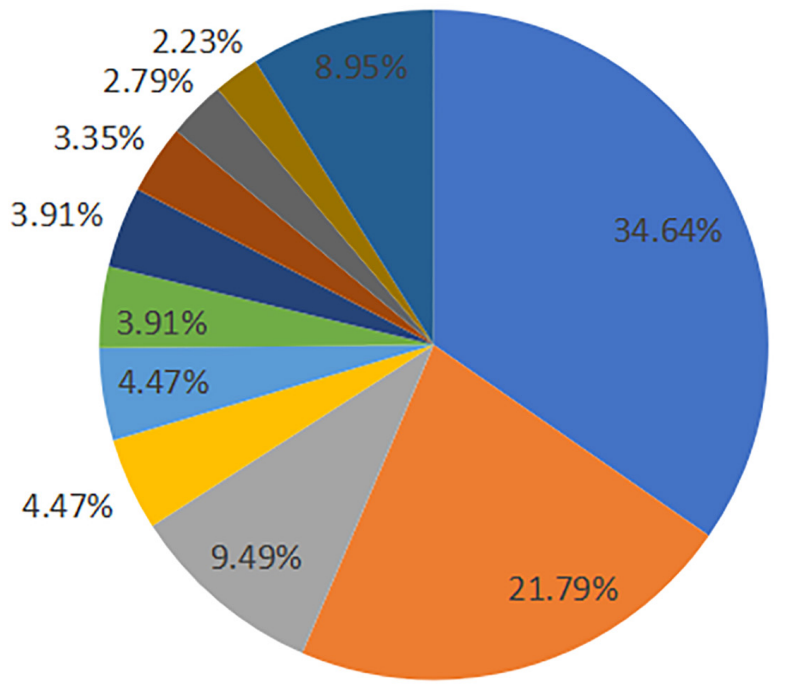

Respiratory

ICU

Hepatology

Neurology

Nephrology

Gastroentererolgy

General Surgery

Neurosurgery

Cardiology

Neonatology

Endocrinology

FIGURE 1 | Distribution of isolated fungi strains in clinic departments. A total of 179 fungal strains were isolated from clinical patients with systemic internal diseases (49 females and 130 males). Of those, 34.64\% were from the respiratory department, $21.79 \%$ were from the intensive care unit, $9.50 \%$ were from the hepatology department, $8.95 \%$ were from the endocrinology department, and $4.47 \%$ were from the neurology department and nephrology department separately.

TABLE 1 | Susceptibility system analysis of invasive fungi infection in clinical patients with systemic internal diseases.

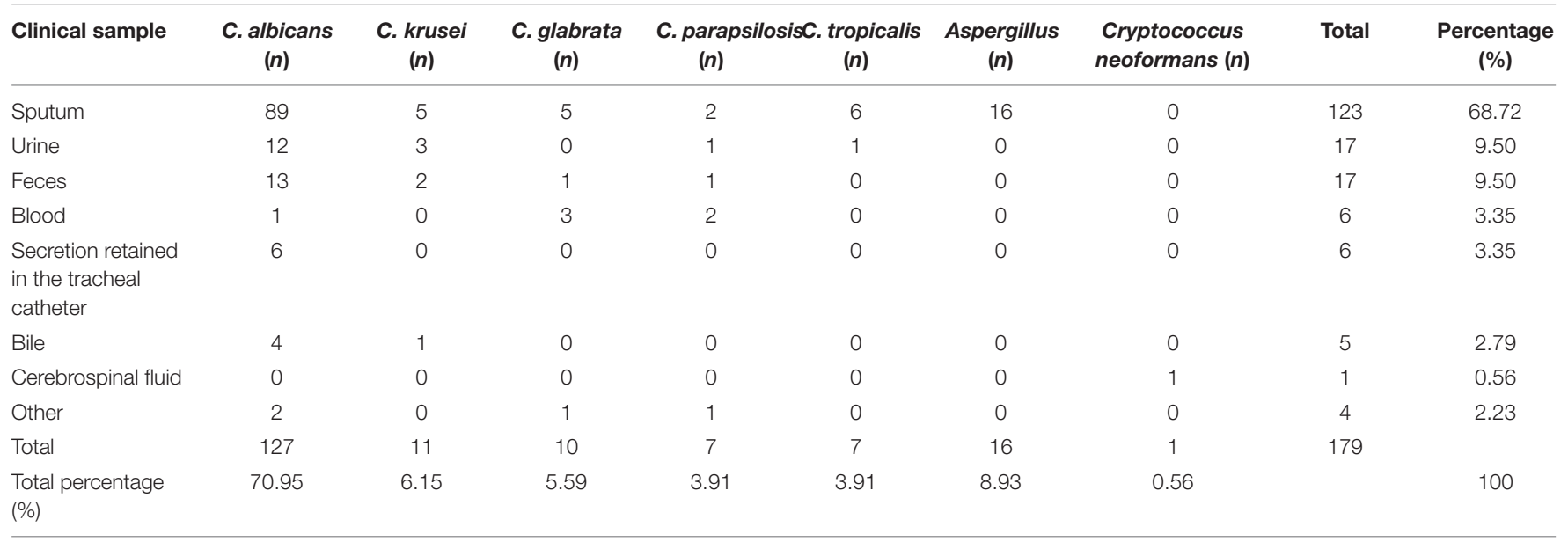

TABLE 2 | In vitro susceptibility among non-Aspergillus fungi.

\begin{tabular}{|c|c|c|c|c|c|c|c|c|c|c|c|c|c|c|c|c|}
\hline Fungal species & Number & \multicolumn{3}{|c|}{ Fluconazole } & \multicolumn{3}{|c|}{ Itraconazole } & \multicolumn{3}{|c|}{ Voriconazole } & \multicolumn{3}{|c|}{$5-\mathrm{FC}$} & \multicolumn{3}{|c|}{ Amphotericin B } \\
\hline C. albicans & 127 & 97.64 & 2.36 & 0 & 97.64 & 1.57 & 0.79 & 100 & 0 & 0 & 98.43 & 0 & 1.57 & 100 & 0 & 0 \\
\hline C. krusei & 11 & 0 & 0 & 100 & 54.55 & 45.45 & 0 & 81.82 & 0 & 18.18 & 72.73 & 27.27 & 0 & 100 & 0 & 0 \\
\hline C. glabrata & 10 & 20.00 & 80.00 & 0 & 20.00 & 80.00 & 0 & 100 & 0 & 0 & 100 & 0 & 0 & 100 & 0 & 0 \\
\hline C. parapsilosis & 7 & 100 & 0 & 0 & 100 & 0 & 0 & 100 & 0 & 0 & 100 & 0 & 0 & 100 & 0 & 0 \\
\hline
\end{tabular}


In this study, 179 strains of invasive fungi were identified and detected. Candida was the main pathogen causing invasive fungal infection in patients with systemic internal diseases. Among the Candida species infecting those patients, Candida albicans was the most frequently recognized, followed by C. krusei, C. glabrata, C. parapsilosis, and C. tropicalis. The rate of C. glabrata infection increased when comparing the patients with sepsis (Bloos et al., 2013).

The antifungal antibiotics were categorized into different groups including azoles, polyenes, fluoropyrimidine analogs, echinocandins, morpholines, allylamines, thiocarbamates, and 5FC. In this study, we analyzed the sensitivity of 163 strains of non-Aspergillus fungi to five antifungal drugs commonly used in clinics. The results of the drug sensitivity testing showed that all of the invasive fungi in this study were $100 \%$ sensitive to amphotericin $\mathrm{B}$, and over $70 \%$ of invasive fungi were sensitive for 5 -FC (only $1.57 \%$ C. albicans with drug resistance). This suggested that amphotericin B and 5-FC should be considered more in clinical treatment (Ramana et al., 2013).

Enoch et al. declared that the epidemiology of Candida infections has changed in the last decade, with a gradual shift from $C$. albicans to non-albicans candida strains that may be less susceptible to azoles (Enoch et al., 2017). The sensitivity of the invasive fungi in this clinical study showed that there was a great difference in sensitivity to azoles. The resistance rate of C. tropicalis for both fluconazole and itraconazole was $41.43 \%$. Moreover, C. albicans and C. parapsilosis were relatively sensitive to azoles. When compared with C. albicans and C. parapsilosis, C. krusei, C. glabrata, and C. tropicalis were less susceptible to fluconazole and itraconazole. The resistance rate of C. krusei for fluconazole was $100 \%$, due to natural resistance. These data have high potential to be useful for the selection of antifungal drugs in the clinical setting.

Combination therapy with amphotericin B and azoles was recommended in cases of localized infection such as meningitis, osteomyelitis, and intra-abdominal infections (OstroskyZeichner, 2008), but our data found that the susceptibility of invasive fungi to zolium drugs presented a genus difference. The frequent use of fluconazole and itraconazole in treating invasive fungi might be the cause of the increase in drug resistance. Badiee and Hashemizadeh (2014) also suggested that monitoring of drug dose was necessary to ensure that therapeutic levels are achieved for optimal clinical efficacy in order to prevent opportunistic invasive fungal infections in sensitive patients. Considering the safety of patients with systemic diseases, a systematic combination of antifungal drugs might increase the incidence of adverse reactions and complications.

There are several limitations to the study. The study population consisted of patients in South China and may not be representative of patients in the general population. Investigation

\section{REFERENCES}

Anaissie, E. J., McGinnis, M. R., and Pfaller, M. A. (2009). Clinical Mycology. Amsterdam: Elsevier Health Science. of multiple fungal infections in patients with severe systemic diseases should also be a concern. Although this is a regional study, we hope that the data could further help multi-regional and worldwide epidemiological surveys, which could provide more favorable clinical evidence for the prevention and treatment of invasive fungal infection in patients with systemic internal diseases in the future.

In this study, we found that patients in the respiratory department, ICU, and hepatology department presented high rates of invasive fungal infections and should include special attention during clinical treatment. The respiratory tract, urinary tract, and gastrointestinal tract were the susceptible systems. Candida, especially C. albicans, was the main pathogen. From the perspective of drug sensitivity, amphotericin B should be given priority in treating the non-Aspergillus fungi infection in patients with systemic internal diseases, while the susceptibility of cynical strains to azoles was variant. These data might provide clinical evidence for the prevention and treatment of invasive fungal infection in patients with systemic internal diseases.

\section{DATA AVAILABILITY STATEMENT}

The raw data supporting the conclusions of this article will be made available by the authors, without undue reservation.

\section{ETHICS STATEMENT}

The studies involving human participants were reviewed and approved by the Clinical Research Ethics Committee and Animal Care and Use Committee (IACUC) of The Third Affiliated Hospital of Sun Yat-sen University-Yuedong Hospital. Written informed consent to participate in this study was provided by the participants' legal guardian/next of kin.

\section{AUTHOR CONTRIBUTIONS}

All authors listed have made a substantial, direct and intellectual contribution to the work, and approved it for publication.

\section{FUNDING}

This research was supported by the Guangdong Basic and Apply Basic Research Foundation (2020A1515010305), National Natural Science Foundation of China (81673085), and Meizhou Science and Technology Program [191224092055033(2019B24)].

Badiee, P., and Hashemizadeh, Z. (2014). Opportunistic invasive fungal infections: diagnosis and clinical management. Indian J. Med. Res. 139, 195-204.

Bassetti, M., Garnacho-Montero, J., Calandra, T., Kullberg, B., Dimopoulos, G., Azoulay, E., et al. (2017). Intensive care medicine research agenda on invasive 
fungal infection in critically ill patients. Intensive Care Med. 43, 1225-1238. doi: 10.1007/s00134-017-4731-2

Bloos, F., Bayer, O., Sachse, S., Straube, E., Reinhart, K., and Kortgen, A. J. J. O. C. C. (2013). Attributable costs of patients with candidemia and potential implications of polymerase chain reaction-based pathogen detection on antifungal therapy in patients with sepsis. J. Crit. Care 28, 2-8. doi: 10.1016/ j.jcrc.2012.07.011

Bonduel, M., Santos, P., Turienzo, C. F., Chantada, G., and Paganini, H. (2001). Atypical skin lesions caused by Curvularia sp. and Pseudallescheria boydii in two patients after allogeneic bone marrow transplantation. Bone Marrow Transplant. 27, 1311-1313. doi: 10.1038/sj.bmt.1703072

Enoch, D. A., Yang, H., Aliyu, S. H., and Micallef, C. (2017). The changing epidemiology of invasive fungal infections. Methods Mol. Biol. 1508, 17-65. doi: 10.1007/978-1-4939-6515-1_2

Ibáñez-Martínez, E., Ruiz-Gaitán, A., and Pemán-García, J. J. R. E. D. Q. (2017). Update on the diagnosis of invasive fungal infection. Rev. Esp. Quimioter. 30(Suppl. 1), 16-21.

Ostrosky-Zeichner, L. (2008). Combination antifungal therapy: a critical review of the evidence. Clin. Microbiol. Infect. 14(Suppl. 4), 65-70. doi: 10.1111/j.14690691.2008.01983.x
Paramythiotou, E., Frantzeskaki, F., Flevari, A., Armaganidis, A., and Dimopoulos, G. J. M. (2014). Invasive fungal infections in the ICU: how to approach, how to treat. Molecule 19, 1085-1119. doi: 10.3390/molecules19011085

Ramana, K. V., Kandi, S., Bharatkumar, V., Sharada, C. V., Rao, R., Mani, R., et al. (2013). Invasive fungal infections: a comprehensive review. Am. J. Infect. Dis. Microbiol. 1, 64-69. doi: 10.12691/ajidm-1-4-2

Smith, J. A., and Kauffman, C. A. J. R. (2012). Pulmonary fungal infections. Respirology 17, 913-926. doi: 10.1111/j.1440-1843.2012.02150.x

Conflict of Interest: The authors declare that the research was conducted in the absence of any commercial or financial relationships that could be construed as a potential conflict of interest.

Copyright $\odot 2021$ Zeng, Peng, Liu, Huang, Zhang, Wen, Lai and Zheng. This is an open-access article distributed under the terms of the Creative Commons Attribution License (CC BY). The use, distribution or reproduction in other forums is permitted, provided the original author(s) and the copyright owner(s) are credited and that the original publication in this journal is cited, in accordance with accepted academic practice. No use, distribution or reproduction is permitted which does not comply with these terms. 